\title{
Impaired Bidirectional Synaptic Plasticity and Procedural Memory Formation in Striatum-Specific cAMP Response Element-Binding Protein-Deficient Mice
}

\author{
Christopher Pittenger, ${ }^{1 \star}$ Stefania Fasano, ${ }^{3,4 \star}$ David Mazzocchi-Jones, ${ }^{5}$ Stephen B. Dunnett, ${ }^{5}$ Eric R. Kandel, ${ }^{1,2}$ and \\ Riccardo Brambilla ${ }^{3}$ \\ ${ }^{1}$ Center for Neurobiology and Behavior and ${ }^{2}$ Howard Hughes Medical Institute, Columbia University College of Physicians and Surgeons, New York, New \\ York 10032, ${ }^{3}$ San Raffaele Scientific Institute, 20132 Milano, Italy, ${ }^{4}$ Istituto di Psicologia, Facoltà di Medicina, Università degli Studi di Milano, 20134 \\ Milano, Italy, and ${ }^{5}$ School of Biosciences, Cardiff University, Cardiff CF10 3US, United Kingdom
}

The striatum has a well documented role in procedural learning and memory. However, the synaptic and molecular mechanisms of acquisition and storage of this form of memory remain poorly understood. We examined procedural memory and plasticity in transgenic mice reversibly expressing a dominant-negative cAMP response element-binding protein (CREB) mutant in the dorsal striatum. In these transgenic mice, corticostriatal long-term potentiation and depression are abolished, indicating that CREB function is essential for bidirectional long-term synaptic plasticity in this structure. Importantly, CREB-deficient animals show reversible alterations in several forms of striatum-dependent memory, including footshock avoidance learning and "response" learning in the cross maze. These findings implicate transcriptional regulation by CREB family transcription factors in striatum-dependent information processing and provide the first clear correlation between procedural learning and memory and synaptic plasticity at the corticostriatal synapse.

Key words: striatum; memory formation; learning and memory; transgenic; synaptic plasticity; genetics

\section{Introduction}

The mammalian nervous system contains multiple learning systems, which can be experimentally isolated from one another and are specialized for different behavioral contexts (Eichenbaum and Cohen, 2001; White and McDonald, 2002). In particular, the dorsal portion of the striatum is involved in motor control and certain forms of procedural and instrumental learning, including stimulus-response (S-R) associations, skill acquisition, and habit formation, whereas its ventral extension, including the nucleus accumbens, is believed to be implicated in the reward mechanisms (White, 1997; Hyman and Malenka, 2001; Packard and Knowlton, 2002; Chao and Nestler, 2004). Ventral and dorsal striatum likely compose an integrated functional structure and are implicated in a variety of diseases, including obsessivecompulsive disorders and addiction (Voorn et al., 2004; Wise, 2004; Everitt and Robbins, 2005).

Transcription factors of the cAMP response element-binding

Received Dec. 19, 2005; revised Jan. 27, 2006; accepted Jan. 27, 2006.

This work was supported by National Institute of Mental Health National Research Service Award MH12956-01, the Columbia University Medical Scientist Training Program (C.P.), the Howard Hughes Medical Institute (E.R.K.), the Medical Research Council UK (S.B.D.), the Italian Ministry of University and Research, the Mariani Foundation for Neurological Research, and the Michael J. Fox Foundation for Parkinson's Research (R.B.).

${ }^{*}$ C.P. and S.F. contributed equally to this work.

Correspondence should be addressed to Riccardo Brambilla, San Raffaele Scientific Institute, Via Olgettina 58 , 20132 Milano, Italy. E-mail: brambilla.riccardo@hsr.it.

C. Pittenger's present address: Department of Psychiatry, Yale University School of Medicine, New Haven, CT 06519.

DOI:10.1523/JNEUROSCI.5406-05.2006

Copyright $\odot 2006$ Society for Neuroscience $\quad$ 0270-6474/06/262808-06\$15.00/0 protein (CREB) family, and the signaling cascades that regulate them, have been found to importantly contribute to memory consolidation both in invertebrates and in several areas of the mammalian brain. In the hippocampus, there is good evidence that CREB also participates in the protein synthesis-dependent late phase of synaptic plasticity, providing a plausible causal link between CREB-mediated transcriptional regulation, dynamic modulation of synaptic strength, and memory consolidation (Barco et al., 2003; Carlezon et al., 2005).

Stimulation of corticostriatal afferents, which can induce synaptic plasticity at this synapse, leads to activation of various transcription factors, including CREB (Sgambato et al., 1998). However, no studies have so far examined the role of CREB family transcription factors in dorsal striatum-dependent synaptic plasticity and procedural memory formation. We explored this possible role in transgenic mice.

\section{Materials and Methods}

Subjects

Generation of str-KCREB transgenic mice has been described previously (Pittenger et al., 2002). str-KCREB mutants and littermate controls were backcrossed for more than seven generations onto C57BL/6. For reversal experiments, 8- to 10-week-old males received doxycycline (dox) at 40 $\mathrm{mg} / \mathrm{kg}$ in food (Bio-Serv, Frenchtown, NJ) for 2 weeks before the beginning of the experiment.

\section{Characterization of transgene expression}

The KCREB expression pattern was visualized by oligonucleotide in situ hybridization as described previously (Pittenger et al., 2002). Western blotting was performed using Trizol extracts of dissected mouse striata 
A

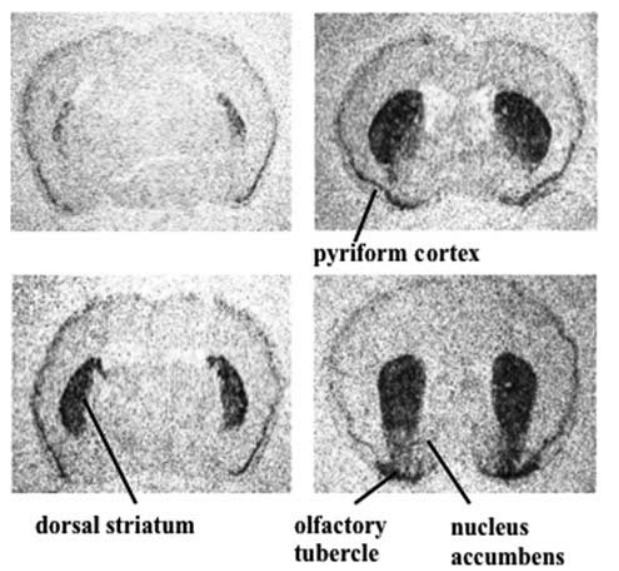

B

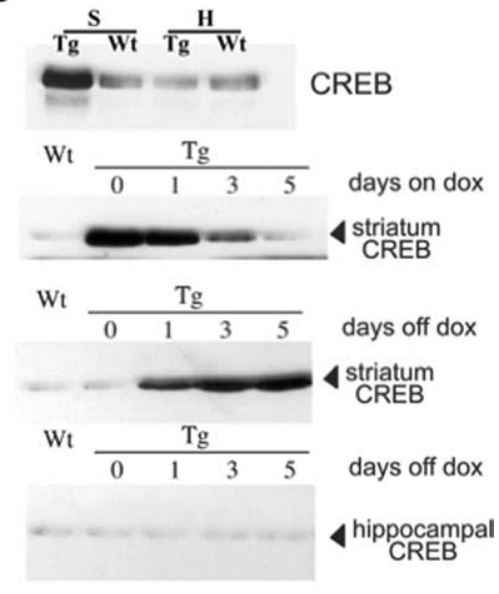

Figure 1. KCREB expression and regulation in transgenic mice. $A$, In situ hybridization shows KCREB predominantly in dorsal striatum. $\boldsymbol{B}$, Total KCREB plus CREB immunoreactivity in striatal (S) and hippocampal (H) extracts. Transgene expression is regulated by dox in food. Tg, trangenic; Wt, Wild type.

was placed in the lighted compartment. As soon as the mouse entered the dark compartment, two consecutive footshocks $(0.2 \mathrm{~mA})$ were delivered with an intershock interval of $2 \mathrm{~s}$. The time spent in the lighted side (step-through latency) on a subsequent exposure to the apparatus is the measure of "inhibitory avoidance." No differences in nociception were detected among groups.

\section{Data analysis}

For electrophysiological and behavioral experiments, statistical comparisons were made by using two-way ANOVA with genotype and time as factors of variation. Subsequent oneway ANOVAs were calculated after significant main effects of the two-way ANOVA. Scheffé test was used when post hoc comparison was required. In the case of the cross maze, $\chi^{2}$ analysis was performed on probe trial data. In all cases, significance was set at $p<0.05$. Analysis was performed with SPSS. and hippocampi probed with rabbit polyclonal anti-CREB antibody (1: 1000; Cell Signaling Technology, Danvers, MA).

\section{Slices and recordings}

Mice were killed via cervical dislocation and decapitated. Brains were quickly removed and immersed into ice-cold artificial CSF (aCSF) [composition (in mM): $126 \mathrm{NaCl}, 2.5 \mathrm{KCl}, 2.5 \mathrm{CaCl}_{2}, 1.3 \mathrm{MgSO}_{4}, 1 \mathrm{NaHPO}_{4}$, $26 \mathrm{NaHCO}_{3}$, and 11 D-glucose] constantly bubbled with $95 \% \mathrm{O}_{2} / 5 \% \mathrm{CO}_{2}$ to maintain the $\mathrm{pH}$ at 7.4. Sagittal sections $(400 \mu \mathrm{m})$ were cut with a vibratome and incubated for $1 \mathrm{~h}$ in a room temperature aCSF. Slices were then equilibrated for $5-10 \mathrm{~min}$ in aCSF, at $31 \pm 0.5^{\circ} \mathrm{C}$, before electrode placement. Extracellular field EPSPs (fEPSPs) were sought from the dorsal striatum, nucleus accumbens, or CA1 region of the hippocampus. Baseline stimulation was at $3 \mathrm{~Hz}$. Long-term depression (LTD) was induced in the dorsal striatum using a modified theta burst protocol, comprising six trains, each train consisting of 10 bursts at $5 \mathrm{~Hz}$, and each burst consisting of four pulses at $100 \mathrm{~Hz}$, with a pulse width of $0.4 \mathrm{~ms}$ and an intertrain interval of $20 \mathrm{~s}$. Long-term potentiation (LTP) was induced in the dorsal striatum using the same tetanus paradigm, but in the absence of $\mathrm{Mg}^{2+}$ ions in the aCSF perfusate. Nucleus accumbens LTP was expressed using the same tetanus paradigm, in the presence of $\mathrm{Mg}^{2+}$ ions. Hippocampal LTP was expressed using a theta burst protocol consisting of three trains, each train consisting of 10 bursts at $5 \mathrm{~Hz}$, and each burst consisting of four pulses at $100 \mathrm{~Hz}$, with a pulse width of $0.4 \mathrm{~ms}$ and an intertrain interval of $20 \mathrm{~s}$, in the presence of magnesium.

\section{Behavioral assays: active avoidance}

In a two-way shuttle-box (Ugo Basile, Comerio, Italy), light [conditioned stimulus (CS)] alternately presented in the two compartments preceded a $0.4 \mathrm{~mA}$ electric footshock [unconditioned stimulus (US)] by $5 \mathrm{~s}$ and overlapped it for $5 \mathrm{~s}$. One hundred CS-US pairings were presented daily for $5 \mathrm{~d}$; an "avoidance response" is a trial on which the animal escapes to the opposite compartment after presentation of the CS, before the US onset (Mazzucchelli et al., 2002). There were no differences in nociception between genotypes at this shock intensity.

Cross maze. The maze consisted of four arms measuring $35 \mathrm{~cm}$ long and $6 \mathrm{~cm}$ wide, with transparent high walls (Passino et al., 2002). Animals were maintained at $80-75 \%$ of their free-feeding weight throughout the experiment. During training trials (four trials per day; $30 \mathrm{~s}$ intertrial interval; for 28 consecutive days excepting day 15) the north and west arms were closed; the east arm was baited with a food pellet, and animals were released from the south arm. On probe trials (days 15 and 30), mice were released from the north arm and had access to the unbaited east (scored as place learners) or west arm (scored as response learners).

Inhibitory avoidance. The inhibitory avoidance apparatus consisted of a small strongly illuminated compartment and a large dark compartment (Ugo Basile) (Mazzucchelli et al., 2002). On the training day, each mouse

\section{Results}

\section{str-KCREB transgenic expression in the striatum}

We have previously generated several lines of mice reversibly expressing KCREB, a potent dominant-negative inhibitor of CREB family transcription factors, to investigate the role of these transcription factors in synaptic plasticity and memory formation (Pittenger et al., 2002). In str-KCREB transgenic mice, in situ hybridization with a specific oligonucleotide probes demonstrated strong transgene expression in dorsal striatum; expression was moderate in olfactory tubercle, much lower in nucleus accumbens shell, and not detectable in accumbens core, hippocampus, or amygdala (Fig. $1 \mathrm{~A}$ ). Importantly, transgene expression could be regulated with doxycycline administered in food (Fig. $1 \mathrm{~B}$ ). Western blot analysis indicates that $5 \mathrm{~d}$ of doxycycline treatment completely shut down KCREB expression in the striatum, but transgene expression recovered after drug removal. No changes in CREB expression could be detected in the hippocampus at baseline or during doxycycline administration. Thus, str-KCREB transgenic mice allow a specific and regulated interference with CREB function in the striatum.

\section{str-KCREB mice show impaired bidirectional corticostriatal synaptic plasticity in the dorsal striatum}

Memory traces are likely to be formed through changes in synaptic strength. LTP and LTD are commonly used to probe the cellular events underlying this information storage (Martin et al., 2000; Malenka and Bear, 2004). However, the relationship of synaptic plasticity in the striatum to its mnemonic functions has rarely been addressed (Mazzucchelli et al., 2002). We therefore examined synaptic plasticity in str-KCREB mice.

We first tested corticostriatal LTD by recording field potentials in acute slices of mouse brain (Calabresi et al., 1992b). Wildtype and str-KCREB mice showed identical basal synaptic transmission as demonstrated by an input/output curve determined across a range of input current (Fig. 2A). However, slices from str-KCREB mice stimulated with a theta burst stimulation (TBS) protocol showed markedly reduced LTD relative to those from sibling controls (Fig. 2B) (two-way ANOVA; time-genotype interaction; $\left.F_{(1,9)}=393.35 ; p<0.0001\right)$. Subsequent one-way ANOVA confirmed that during the $60 \mathrm{~min}$ poststimulus period, str-KCREB slices did differ from the baseline ( post hoc Scheffé test; $p<0.0001$ ) but only showed a transient, short-term form of 
A Input/output curve in dorsal striatum

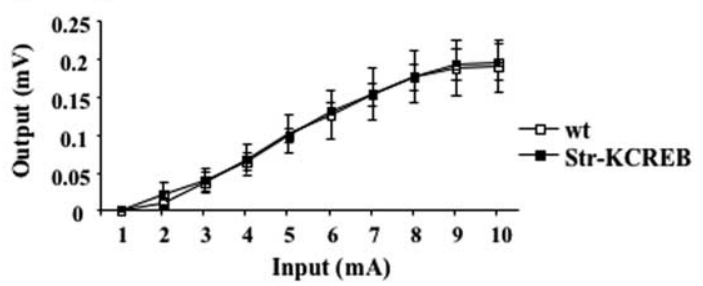

B LTD in Dorsal Striatum
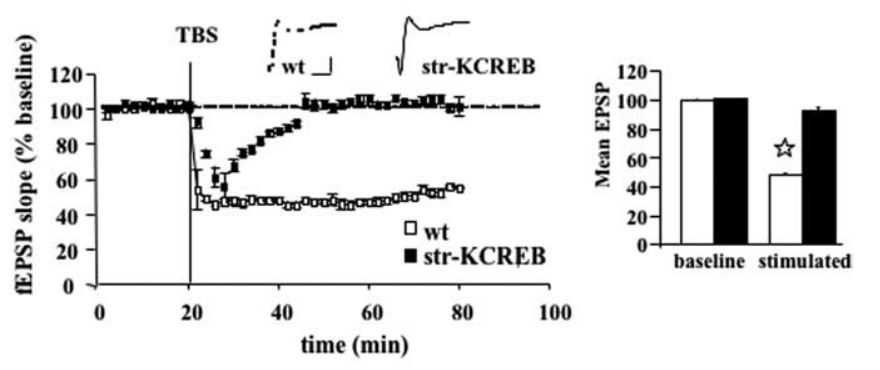

C LTP in Dorsal Striatum

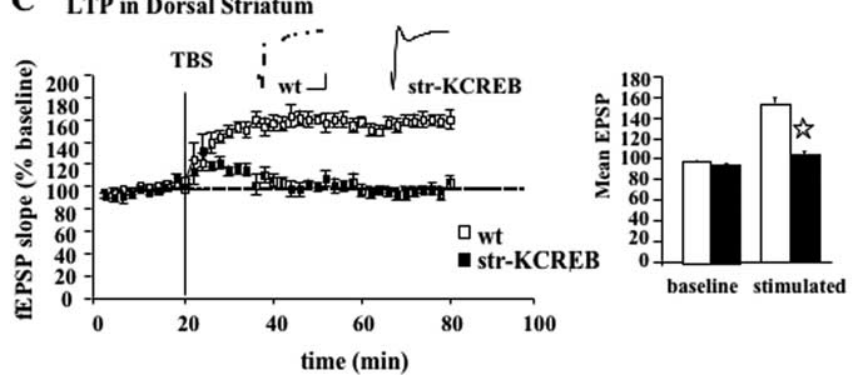

Figure 2. Loss of bidirectional synaptic plasticity in dorsal striatum of str-KCREB mice. $\boldsymbol{A}$, str-KCREB mice have normal basal corticostriatal synaptic transmission, as shown by an input/ output curve of wild-type $(n=7)$ and transgenic $(n=8)$ slices, measured in the presence of 30 $\mu$ m bicuculline. $\boldsymbol{B}$, str-KCREB mouse slices $(n=5)$ show impaired corticostriatal theta burst (TBS)-induced LTD in comparison with wild-type controls $(n=6)$. C, str-KCREB mouse slices $(n=5)$ show impaired corticostriatal LTP in comparison with wild-type controls $(n=6)$. Open star, Genotype effect, $p<0.0001$. Sample baseline traces of wild-type and transgenic mice are shown in $\boldsymbol{B}$ and $\boldsymbol{C}$, left panels. Calibration: $1 \mathrm{mV}, 2 \mathrm{~ms}$. Left panels, Time course comprising 20 min baseline and $60 \mathrm{~min}$ after theta burst stimulation. Right panels, Average of $60 \mathrm{~min}$ after stimulus. wt, Wild type. Error bars indicate SEM.

plasticity. In fact, whereas wild-type slices produced LTD (48.1 $0.89 \%$ of the baseline, for the entire 60 min poststimulus time period), str-KCREB slices failed to do so $(92.6 \pm 1.5 \%$ of the baseline; str-KCREB vs wild-type; 60 min poststimulus period; post hoc Scheffé test, $p<0.0001)$.

In the absence of extracellular magnesium, the same theta burst stimulus (which now activates NMDA glutamate receptors more strongly) induces LTP at these synapses (Calabresi et al., 1992a). We found LTP induction in the str-KCREB mutants also to be severely impaired (Fig. $2 C$ ). Two-way ANOVA indicated a significant time-genotype interaction $\left(F_{(1,8)}=78.0 ; p<0.0001\right)$. Post hoc comparisons showed no statistically significant differences between the $20 \mathrm{~min}$ baseline and $60 \mathrm{~min}$ poststimulus period in str-KCREB mice $(107.7 \pm 4.0 \%$ of the baseline, for the entire 60 min poststimulus time period; Scheffé test, $p=0.83$ ), whereas poststimulus LTP in wild-type animals was found significantly different from all other conditions $(159.5 \pm 6.1 \%$ of the baseline, for the entire $60 \mathrm{~min}$ poststimulus time period; Scheffé test, $p<0.0001$ ).

In situ hybridization data (Fig. $1 \mathrm{~A}$ ) demonstrated that trans-

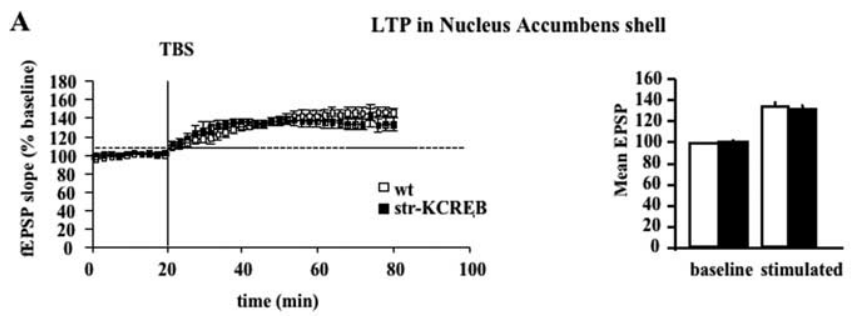

B
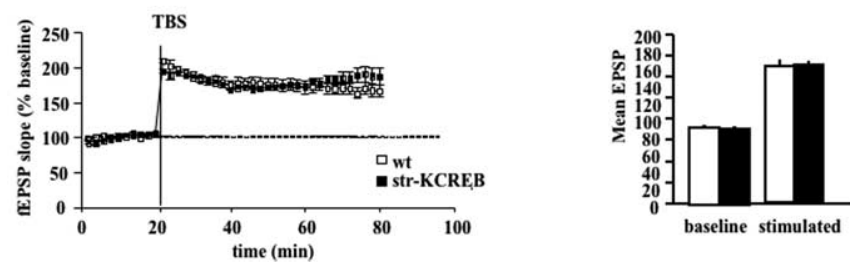

Figure 3. Normal synaptic plasticity in nucleus accumbens and hippocampus of str-KCREB mice. $A$, TBS in the shell of the nucleus accumbens ( $n=8$ transgenics; $n=8$ wild type). $B$, TBS at the CA3-CA1 Schaffer collateral synapse ( $n=5$ transgenics; $n=6$ wild type). wt, Wild type. Error bars indicate SEM.

gene expression is predominantly in the dorsal striatum. However, this analysis is of limited sensitivity, and even low levels of expression in ventral striatal structures such as the nucleus accumbens shell could, in principle, perturb CREB function enough to have a physiological effect. Analysis of synaptic plasticity is a more appropriate probe of the physiological consequences of transgene expression at the functional level. We therefore examined corticostriatal plasticity in the accumbens shell. Corticostriatal LTP can be readily elicited in the ventral striatum, although similarities and differences between the molecular mechanisms of LTP in dorsal and ventral striatum remain mostly unresolved (Fasano and Brambilla, 2002). A TBS protocol identical to that used for eliciting LTD in the dorsal striatum induces LTP in the accumbens shell, at physiological levels of magnesium. We found that corticostriatal LTP in accumbens slices from strKCREB mice was indistinguishable from that seen in sibling controls (60 min poststimulus means in wild-type, $134.3 \pm 4.8 \%$; in str-KCREB, $132.5 \pm 4.0 \%$ ) (Fig. $3 A$ ). As a final control, we induced LTP at the CA3-CA1 Schaffer collateral synapse (Fig. 3B). As expected, given the absence of transgene expression in the hippocampus, we found normal hippocampal LTP in this structure (poststimulus mean responses in wild-type, $178.8 \pm 6.7 \%$; in str-KCREB, $180.7 \pm 3.3 \%$ ).

In conclusion, we found a specific impairment in bidirectional synaptic plasticity in the dorsal portion of the striatum. This finding supports the notion that CREB function in the dorsal portion of the striatum has a crucial role in both LTP and LTD.

\section{Deficits in multiple trial procedural learning tasks in str-KCREB mice}

The striatum is involved in various forms of instrumental conditioning, a type of procedural memory. In particular, the dorsal striatal circuitry is thought to be essential for S-R associations that tend to become automated over time and are thought to be encoded in procedural memory networks (Eichenbaum and Cohen, 2001; Packard and Knowlton, 2002). To test the hypothesis that CREB inhibition in the dorsal striatum of str-KCREB mice may affect memory formation, we first applied behavioral tests characterized by $\mathrm{S}-\mathrm{R}$ associations acquired through multiple repetitions. 
A Active avoidance
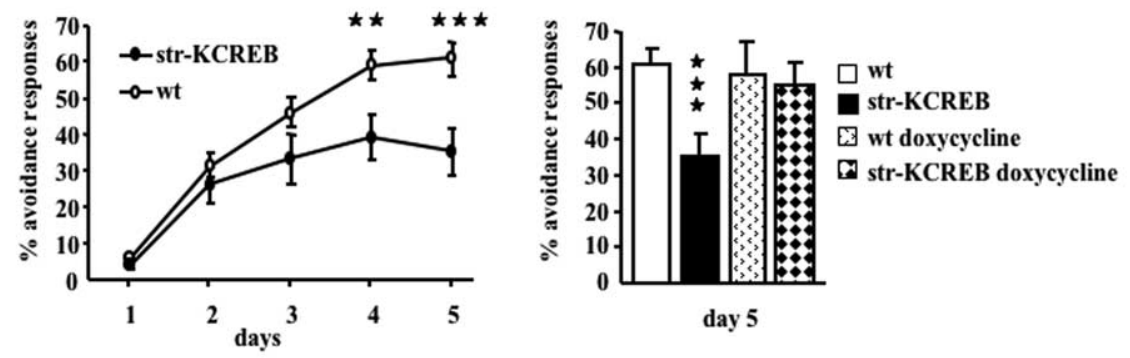

B Cross-Maze

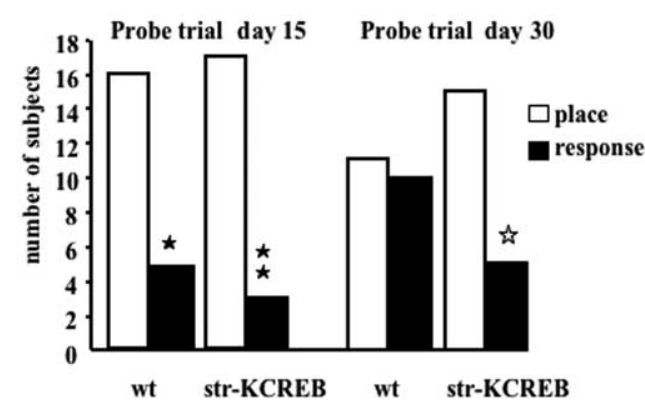

Figure 4. Impaired procedural memory in str-KCREB mice. $A$, Active avoidance. Left panel, str-KCREB mice show impaired avoidance of shock (100 trials per day; $5 \mathrm{~d}$ ). Two filled stars, $p<0.01$; three filled stars, $p<0.001$. Right panel, Performance on day 5 [30 wild type (wt), 23 str-KCREB, 13 wt plus dox, 13 str-KCREB plus dox]. $B$, str-KCREB mice show persistent bias toward a spatial strategy in the cross maze (4 trials per day). Left side, Place versus response strategy at probe trial on day 15 ( $21 \mathrm{wt}, 21$ str-KCREB). Place versus response learning: one filled star, $p<0.05$; two filled stars, $p<0.01$. Right side, Probe trial on day 30. Wild-type versus transgenics: one open star, $p<0.05$. C, str-KCREB mice show normal learning and memory at $1 \mathrm{~h}$ but impaired long-term memory at $24 \mathrm{~h}$ in an inhibitory-avoidance task. Inhibitory-avoidance learning as measured by step-through latency of str-KCREB and control animals, tested at $1 \mathrm{~h}$ ( 12 wild type, 12 str-KCREB) and $24 \mathrm{~h}$ ( 30 wild type, 30 str-KCREB, without dox; 12 wild type, 12 str-KCREB with dox) (all values are mean \pm SEM). Four filled stars, $p<0.0001$.

The active avoidance paradigm is conducted in a shuttle box (Mazzucchelli et al., 2002). Over multiple trials, animals learn to associate a cue (a light) with an incipient shock and to avoid the shock by escaping to a neighboring chamber. Acquisition of this task has been shown through lesion studies to depend on the dorsal striatum, although other structures, including the nucleus accumbens and the amygdala, also participate (Schutz and Izquierdo, 1979) (for review, see Fasano and Brambilla, 2002).

str-KCREB animals showed attenuated learning in this task, relative to littermate controls (Fig. $4 A$, left panel). Two-way ANOVA for repeated measures showed a significant day-genotype interaction $\left(F_{(1,51)}=11.77 ; p<0.001\right)$. Transgenic animals avoided the cued shock less often than littermate controls (oneway ANOVA; $F_{(1,51)}=7.47, p<0.01$ on day $4 ; F_{(1,51)}=11.48$, $p<0.001$ on day 5). However, when transgene expression was turned off with doxycycline in adult animals before training, strKCREB animals and littermate controls performed equivalently (Fig. $4 A$, right panel).

Although active avoidance depends on the striatum and is acquired over multiple trials, it is a problematic task to interpret, because it also depends on multiple other cortical and subcortical structures. It also requires a potent aversive stimulus, a footshock. We therefore investigated the performance of str-KCREB mice in another test, the cross maze, in which the relative contribution of the dorsal striatum is unambiguous (Packard and McGaugh, 1996) (for review, see Packard and Knowlton, 2002). In this task, animals learn to locate a food reward, placed in the east arm, using either a "place" strategy, which is hippocampus dependent, or a "response" strategy, which is striatum dependent.
The strategies are dissociated during a probe trial in which the start location is rotated $180^{\circ}$ (from the south to the north arm), such that the two strategies will yield opposite search behaviors. We predicted that, if str-KCREB mice are deficient in striatum-dependent learning, they should be biased toward the spatial strategy.

We tested str-KCREB mice and littermate controls in a version of this task modified for mice, which show relatively little procedural learning in the task as originally described (Passino et al., 2002). In our protocol, both north and west arms were closed during training, to facilitate the formation of procedural memory. After $14 \mathrm{~d}$ of such training (probe trial at day 15), both transgenic and wild-type mice showed a marked preference for a "place" strategy, without differences between genotypes, consistent with intact hippocampal circuitry in the strKCREB mutant mice (Fig. $4 B$, left panel) (wild-type vs transgenics: $\chi^{2}=0.86 ; p=$ $0.35)$. However, after an additional $14 \mathrm{~d}$ of continuous training, a second probe trial (day 30 ) showed that $48 \%$ of wild-type animals now used a "response" learning strategy, whereas str-KCREB mice remained strongly biased toward the place strategy (Fig. 4B, right panel) (wild-type vs transgenics: $\left.\chi^{2}=3.95 ; p<0.05\right)$.

Together, these results demonstrate that str-KCREB mice manifest profound deficits in two forms of striatum-dependent procedural learning that rely on distinct stimuli and behavioral outputs.

\section{Long-term procedural memory is impaired in str-KCREB mice}

Active avoidance and response learning in the cross maze share the property of being acquired over many training trials. Although this characteristic makes them good models of procedural learning, which is often a gradual process, it renders it impossible to dissociate initial learning from consolidation of learned information and long-term memory. In other studies of transcription factors thought to be involved in memory consolidation, it has been possible to test animals in tasks in which learning occurs in a single trial, such as fear conditioning. This permits testing at different defined intervals after training, allowing dissociation of learning from long-term memory. We sought an analogous way to make this dissociation in striatumdependent learning str-KCREB mice.

Inhibitory avoidance learning depends on multiple cortical and subcortical structures, including both dorsal and ventral striatum as well as hippocampus and amygdala (Prado-Alcala et al., 1975) (for review, see Fasano and Brambilla, 2002). Because learning occurs in a single trial test, and despite some interpretive difficulties shared with active avoidance, it allows dissociation of effects on short- and long-term memory. Animals are placed in a lighted arena with free access to a darkened compartment. When they enter the dark compartment, they receive an aversive footshock. Their subsequently increased latency to enter the darkened compartment is a measure of their learning the association 
between that chamber and the aversive stimulus (Mazzucchelli et al., 2002).

str-KCREB mice learned this task equivalently to sibling controls: both groups showed significantly increased latency $1 \mathrm{~h}$ after training (two-way ANOVA learning effect; $F_{(1,22)}=241.45 ; p<$ 0.0001) with no significant difference between them (Fig. 4C) (genotype effect; $F_{(1,22)}=0.94 ; p=0.34$ ). However, in a separate cohort of animals tested $24 \mathrm{~h}$ after pairing, str-KCREB animals showed significantly impaired memory relative to sibling controls (two-way ANOVA genotype effect; $F_{(1,58)}=33.13 ; p<$ $0.0001)$, and relative to the performance of str-KCREB animals at $1 \mathrm{~h}(p<0.01)$. When transgene expression was turned off with doxycycline before training (Fig. $1 B$ ), a third group of mutant animals remembered normally at $24 \mathrm{~h}$ (Fig. $4 C$ ).

These data indicate that the behavioral impairment associated with CREB inhibition in the dorsal striatum is likely linked to the inability of these mice to consolidate procedural memories. This effect may also underlie the impaired performance in more gradually acquired paradigms such as active avoidance and the cross maze.

\section{Discussion}

In sum, we have been able to attenuate the function of CREB family transcription factors in dorsal striatum through expression of a dominant-negative CREB mutant, KCREB. These animals have a specific deficit in distinct forms of striatumdependent procedural learning, without showing deficits in either motor learning on the rotarod or in spatial learning in the Morris water maze (data not shown). Furthermore, the mutant animals show a marked deficit in both LTP and LTD at the glutamatergic corticostriatal projection.

Whereas the role of the dorsal striatum in cognitive processes is increasingly appreciated, the cellular and molecular mechanisms of striatum-dependent procedural learning are poorly understood. Although corticostriatal synaptic plasticity has been extensively investigated, its relationship to striatum-dependent procedural learning remains unclear.

One obstacle to clarifying this relationship has been the difficulty of identifying clearly dorsal striatum-dependent learning tasks. This contrasts, for example, with the clear role of the hippocampus in spatial learning in the Morris water maze and of the amygdala in fear conditioning. The attenuation of active avoidance learning, for example, could result from alterations of any of several learning-related subcortical structures and thus would be difficult to interpret in isolation. In this context, our finding of a response-learning deficit in the cross maze is of particularly importance. This task is critically regulated by the dorsal striatum: dorsal striatal lesions or reversible inactivation impair the procedural learning component, whereas infusion of glutamate or other neuronal activators enhances it (Packard and McGaugh, 1996; Packard, 1999; Yin and Knowlton, 2004).

The specificity of the learning deficit that we observed is further emphasized by the specificity of KCREB transgene expression, which is not found in hippocampus, amygdala, or other structures outside the basal ganglia that might complicate interpretation of behavioral phenotypes. Moreover, the reversibility of the active and inhibitory avoidance phenotypes demonstrates that they do not derive from irreversible consequences of CREB disruption such as neuronal death or alterations in neural development.

The idea that memory changes in these tests are linked to dorsal striatal function is also supported by the electrophysiological data. We see profound deficits in both LTP and LTD at corticostriatal synapses in the dorsal striatum, but not in the accum- bens shell. Multiple, mechanistically overlapping forms of synaptic plasticity can coexist at a single synapse, and it is not clear how to measure LTD or LTP to best model the processes that are involved in vivo in information storage during striatumdependent procedural learning. The deficit in synaptic plasticity that we see in these animals is thus best interpreted conservatively, as showing merely that mechanisms involved in the regulation of synaptic strength are not functioning normally in dorsal striatum. However, the extent of the deficit in bidirectional synaptic plasticity in these standard experimental paradigms is striking, and clearly consistent with a causal link between information storage at this synapse and dorsal striatum-dependent learning.

A number of studies have implicated CREB family transcription factors in various forms of learning and memory, although the generality of the CREB-plasticity-learning linkage beyond the hippocampus remains unclear (for review, see Barco et al., 2003; Carlezon et al., 2005). Even in the hippocampus, for which experimental support for a causal association between CREBmediated transcription and learning is relatively clear, disruption of CREB activity produces a deficit in synaptic plasticity that is remarkably subtle (Pittenger et al., 2002; Huang et al., 2004). Our data provide strong new support for the generality of this causal link to other structures in the mammalian brain.

Together, although no single correlation can indubitably confirm a causal relationship, these experiments provide the first evidence connecting gene transcription, corticostriatal synaptic plasticity, and striatum-dependent procedural learning.

\section{References}

Barco A, Pittenger C, Kandel ER (2003) CREB, memory enhancement and the treatment of memory disorders: promises, pitfalls and prospects. Expert Opin Ther Targets 7:101-114.

Calabresi P, Pisani A, Mercuri NB, Bernardi G (1992a) Long term potentiation in the striatum is unmasked by removing the voltage-dependent blockade of NMDA receptor channel. Eur J Neurosci 4:929-935.

Calabresi P, Maj R, Pisani A, Mercuri NB, Bernardi G (1992b) Long-term synaptic depression in the striatum: physiological and pharmacological characterization. J Neurosci 12:4224-4233.

Carlezon Jr WA, Duman RS, Nestler EJ (2005) The many faces of CREB. Trends Neurosci 28:436-445.

Chao J, Nestler EJ (2004) Molecular neurobiology of drug addiction. Annu Rev Med 55:113-132.

Eichenbaum H, Cohen NJ (2001) From conditioning to conscious recollection: memory systems of the brain. Oxford: Oxford UP.

Everitt BJ, Robbins TW (2005) Neural systems of reinforcement for drug addiction: from actions to habits to compulsion. Nat Neurosci 8:1481-1489.

Fasano S, Brambilla R (2002) Cellular mechanisms of striatum-dependent behavioral plasticity and drug addiction. Curr Mol Med 2:649-665.

Huang YY, Pittenger C, Kandel ER (2004) A form of long-lasting, learningrelated synaptic plasticity in the hippocampus induced by heterosynaptic low-frequency pairing. Proc Natl Acad Sci USA 101:859-864.

Hyman SE, Malenka RC (2001) Addiction and the brain: the neurobiology of compulsion and its persistence. Nat Rev Neurosci 2:695-703.

Malenka RC, Bear MF (2004) LTP and LTD: an embarrassment of riches. Neuron 44:5-21.

Martin SJ, Grimwood PD, Morris RG (2000) Synaptic plasticity and memory: an evaluation of the hypothesis. Annu Rev Neurosci 23:649-711.

Mazzucchelli C, Vantaggiato C, Ciamei A, Fasano S, Porrazzo A, Orban PC, Pakhotin P, Krezel W, Wezl H, Wolfer DP, Pages G, Valverde O, Marowsky A, Maldonado R, Ehrengruber MU, Cestari V, Lipp H-P, Chapman PF, Pouyssegur J, Brambilla R (2002) Knockout of ERK1 MAP kinase enhances synaptic plasticity in the striatum and facilitates striatal-mediated learning and memory. Neuron 34:807-820.

Packard MG (1999) Glutamate infused posttraining into the hippocampus or caudate-putamen differentially strengthens place and response learning. Proc Natl Acad Sci USA 96:12881-12886. 
Packard MG, Knowlton BJ (2002) Learning and memory functions of the basal ganglia. Annu Rev Neurosci 25:563-593.

Packard MG, McGaugh JL (1996) Inactivation of hippocampus or caudate nucleus with lidocaine differentially affects expression of place and response learning. Neurobiol Learn Mem 65:65-72.

Passino E, Middei S, Restivo L, Bertaina-Anglade V, Ammassari-Teule M (2002) Genetic approach to variability of memory systems: analysis of place vs. response learning and fos-related expression in hippocampal and striatal areas of C57BL/6 and DBA/2 mice. Hippocampus 12:63-75.

Pittenger C, Huang YY, Paletzki RF, Bourtchouladze R, Scanlin H, Vronskaya S, Kandel ER (2002) Reversible inhibition of CREB/ATF transcription factors in region CA1 of the dorsal hippocampus disrupts hippocampusdependent spatial memory. Neuron 34:447-462.

Prado-Alcala RA, Grinberg ZJ, Arditti ZL, Garcia MM, Prieto HG, BrustCarmona H (1975) Learning deficits produced by chronic and reversible lesions of the corpus striatum in rats. Physiol Behav 15:283-287.
Schutz RA, Izquierdo I (1979) Effect of brain lesions on rat shuttle behavior in four different tests. Physiol Behav 23:97-105.

Sgambato V, Pages C, Rogard M, Besson MJ, Caboche J (1998) Extracellular signal-regulated kinase (ERK) controls immediate early gene induction on corticostriatal stimulation. J Neurosci 18:8814-8825.

Voorn P, Vanderschuren LJ, Groenewegen HJ, Robbins TW, Pennartz CM (2004) Putting a spin on the dorsal-ventral divide of the striatum. Trends Neurosci 27:468-474.

White NM (1997) Mnemonic functions of the basal ganglia. Curr Opin Neurobiol 7:164-169.

White NM, McDonald RJ (2002) Multiple parallel memory systems in the brain of the rat. Neurobiol Learn Mem 77:125-184.

Wise RA (2004) Dopamine, learning and motivation. Nat Rev Neurosci 5:483-494.

Yin HH, Knowlton BJ (2004) Contributions of striatal subregions to place and response learning. Learn Mem 11:459-463. 